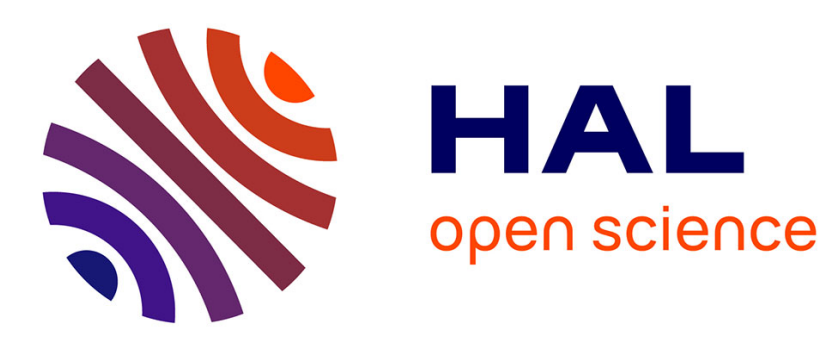

\title{
Blind PDF Document Watermarking Robust Against PCA and ICA Attacks
}

Makram Hatoum, Rony Darazi, Jean Couchot

\section{To cite this version:}

Makram Hatoum, Rony Darazi, Jean Couchot. Blind PDF Document Watermarking Robust Against PCA and ICA Attacks. International Conference on e-business and Telecommunications, Jul 2018, Porto, Portugal. hal-02366766

\section{HAL Id: hal-02366766 https://hal.science/hal-02366766}

Submitted on 16 Nov 2019

HAL is a multi-disciplinary open access archive for the deposit and dissemination of scientific research documents, whether they are published or not. The documents may come from teaching and research institutions in France or abroad, or from public or private research centers.
L'archive ouverte pluridisciplinaire HAL, est destinée au dépôt et à la diffusion de documents scientifiques de niveau recherche, publiés ou non, émanant des établissements d'enseignement et de recherche français ou étrangers, des laboratoires publics ou privés. 


\title{
Blind PDF Document Watermarking Robust Against PCA and ICA Attacks
}

\author{
Makram W. Hatoum ${ }^{1}$, Rony Darazi ${ }^{2}$ and Jean-François Couchot ${ }^{1}$ \\ ${ }^{1}$ FEMTO-ST Institute, University of Bourgogne Franche-Comté, UMR 6174 CNRS, France \\ ${ }^{2}$ TICKET Lab, Antonine University, Hadat-Baabda, Lebanon \\ \{makram.hatoum,jean-francois.couchot $\} @$ univ-fcomte.fr, rony.darazi@ua.edu.lb
}

\begin{abstract}
Keywords: Digital watermarking, Portable Document Format, QIM watermarking, BSS, Security, Transparency, Robustness.

Abstract: $\quad$ Spread Transform Dither Modulation (STDM) is a blind watermarking scheme used for its high robustness against re-quantization and random noise attacks. It has been applied mainly on images, speech, and PDF documents. The key of this scheme is the projection vector aiming at spreading the embedded message over a set of cover elements. However, it has been recently shown that such a key vector can be estimated thanks to Blind Source Separation (BSS) techniques, e.g. Principal Component Analysis (PCA) and Independent Component Analysis (ICA). This security breach can be harnessed by an opponent to copy, remove, or modify the embedded watermark. In this position paper, a CAR-STDM (Component Analysis Resistant-STDM) is designed and its application on PDF documents is presented. The security is guaranteed by the use of a cryptographically secure number generator, preventing algebraic approaches (as BSS ones) from finding the key. First experimental results show that the Secure-STDM achieves the security against the aforementioned BSS techniques. It is further shown that CAR-STDM preserves its robustness against AWGN and Salt\&Peper attacks, and keeps furthermore its transparency.
\end{abstract}

\section{INTRODUCTION}

Digital networks are essential communication mechanisms that are used to transmit any sort of information such as text, audio, and image. The rapid growth of the volume of exchanged data over the internet provides a significant number of problems such as illegal distribution, authentication, duplication and malicious tampering of the digital data. Authors and data providers are concerned about protecting their data or work, to be distributed in a network environment. Among many approaches of protection, digital watermarking is undoubtedly the one that has received the most attention and interest. Watermarking is the practice of transparently, which alter a digital content like image, video, audio, and text, for various purposes, such as copyright protection, authentication, access control, and broadcast monitoring (Cox et al., 2007). The watermarking approaches are classically applied into the spatial domain and frequency domain. In the first one, the watermark is embedded directly into the cover work by changing the intensity values of the signal. In the second one, the watermark is embedded into the frequency domain transform, such as the DCT, DFT, and DWT. Over the last decade, several watermarking schemes have been proposed, which can be classified into the additive class know by Spread Spectrum (SS) schemes (Cox et al., 1996), and the substitutive class known by Quantization Index Modulation (QIM) (Chen and Wornell, 2001a). Spread Transform Dither Modulation (STDM) is an extension of QIM that is more robust against requantization and random noise attacks. With the STDM method, each bit of the watermark is inserted into a sample vector $x$ after quantizing the projection of $x$ onto a projection vector $p$.

The watermarking schemes are characterized by a number of properties such as payload, imperceptibility, robustness, and security. Payload defines the amount of data that can be embedded in the original signal. Imperceptibility refers to the perceptual similarity between the original signal and the watermarked one. Robustness refers to the ability to uncover the watermark after several types of attacks such as additive noise, compression, and filtering. The security constraint has received little attention, while the most of the watermarking schemes have been driven by the improvement of the above require- 
ments. The security relies on the goals and the power of an adversary to copy, modify or remove the watermark. (Bas and Hurri, 2006) show that STDM can be attacked successfully using a Blind Source Separation (BSS) technique called Independent Component Analysis (ICA), by estimating the projection vector $p$ of STDM used during the embedding process. (Cao, 2014) proved that an attacker can estimate the projection vector $p$ using another BSS technique called Principal Component Analysis (PCA). Therefore, they proposed an improved method for STDM called ISTDM in order to resist such kind of BSS attack.

Portable Document Format, a digital form for representing documents, was developed and specified by Adobe Systems Society (Iso, 2008). Several methods have been proposed in PDF and Text documents. (Por and Delina, 2008) presented an approach in information hiding, using inter-paragraph and inter-word spacing, characterized by a large capacity for embedding the hidden bits. This method misses the main constraint of robustness. When deleting the space between words and paragraphs, the hidden data is destroyed. (Wang and Tsai, 2008) proposed an imperceivable modification of PDF object parameters. The main drawback of this method is the low embedding capacity. (Lee and Tsai, 2010) presented two methods to embed the secret messages in a PDF file using the alternative space coding method and the null space coding method. In those methods, The ASCII code 20 (original white space) and the ASCII code A0 (nonbreaking space) are used as the watermarking space. An opponent could simply modify or remove the embedded message by replacing the ASCII code A0 by 20. (Alizadeh-Fahimeh et al., 2012) composed two different algorithms using the TJ method. The first one has a lower capacity level, and the second one has a higher embedding capacity with a lower security level. (Lin et al., 2013) presented a study based on PDF files of iso-8859 encoding. It consists of hiding information without detecting any irregularity while reading the PDF file. The main drawback is that the hiding information is removed when the PDF file is opened directly without using PDF reader. A blind digital watermarking scheme for PDF documents is proposed by (Bitar et al., 2017). This method consists of embedding the secret message in the $x$-coordinates of a group of characters, taking into consideration the transparency-robustness trade-off. (Kuribayashi et al., 2017) used the space lengths between characters as a watermarking space, and the watermark is embedded using the DCT transform, based on the DM-QIM, but this method still missing the experimental tests concerning the robustness constraints.
This position paper introduces the CAR-STDM (Component Analysis Resistant-STDM) for blind PDF watermarking robust against PCA and ICA attacks. This paper recalls some backgrounds on STDM and BSS attacks in Section 2. The proposed method is presented in Section 3. The evaluation of the proposed approach is presented in section 4 . Finally, in Section 5 conclusion and future work are given.

\section{BACKGROUND}

\subsection{Spread Transform Dither Modulation}

QIM methods proposed by (Chen and Wornell, 2001a) use knowledge of the host signal at the encoder, and show an achievable trade-off among the robustness, degradation of the host signal, and the embedding rate. QIM watermarking algorithm quantizes each signal sample $x$, using a quantizer $Q_{m}$, based on the message bit $m \in\{0,1\}$. We are going to take $Q_{0}$ for $m=0$, and $Q_{1}$ for $m=1$.

$$
\begin{aligned}
& Q_{0}(x, \Delta)=\text { round }\left(\frac{x-d_{0}}{\Delta}\right) \Delta+d_{0} \\
& Q_{1}(x, \Delta)=\text { round }\left(\frac{x-d_{1}}{\Delta}\right) \Delta+d_{1}
\end{aligned}
$$

where $\Delta$ : represents the Quantization Factor, round $($.$) : rounding value to the nearest integer,$ $d_{0}$ and $d_{1}$ : real values represent the dither level

$$
d_{0}=-\frac{\Delta}{4} \text { and } d_{1}=\frac{\Delta}{4} \text {. }
$$

STDM is a special case of QIM, it is called Spread Transform Dither Modulation due to the spreading of the embedding-induced distortion into all groups of samples instead of one. Each bit of the secret message is inserted into a sample vector $x$ of length $N$ producing a vector $y$. Instead of quantizing the host signal itself, the quantization occurs entirely in the projection of $x$ onto a projection vector $p$. The quantized signal is given by:

$$
y=x+\left(\operatorname{round}\left(\frac{x^{T} p-d_{m}}{\Delta}\right) \Delta+d_{m}-x^{T} p\right) p
$$

where $x^{T}$ is the transpose of $x$, and $d_{m}$ is defined as in equation (3).

Equation (4) can be seen as the host signal $x$ augmented with the quantization error $q^{\prime}$ :

$$
q^{\prime}=\left(\operatorname{round}\left(\frac{x^{T} p-d_{m}}{\Delta}\right) \Delta+d_{m}-x^{T} p\right) p
$$


To extract the embedded message, the detection can be performed with a minimum distance decoder as the form:

$$
\hat{m}=\underset{m \in\{0,1\}}{\operatorname{argmin}}\left|y^{T} p-Q_{m}\left(y^{T} p, \Delta\right)\right|
$$

\subsection{BSS Techniques}

Beside imperceptibility and robustness, the security constraint is an important requirement for the watermarking schemes. While increasing the security, we guarantee that the embedded watermark is safe against the opponent, whose aim is to estimate the private key. Among Blind Source Separation (BSS) techniques, we focus here on Principal Component Analysis (PCA) and Independent Component Analysis (ICA) recalled hereafter.

\subsubsection{ICA Attack}

An attacker may estimate the projection vector $p$ of STDM using a blind source separation technique called Independent Component Analysis (ICA), which is a computational and statistical technique for recovering a set of independent signals from sets of random variables or signals by some simple assumptions of their statistical properties (Hyvarinen, 1999; Hyvärinen et al., 2004).

In ICA, the measure is based on non-gaussianity, and this is according to the central limit theorem; the average of independent variables will have a distribution that is closer to Gaussian, and the mixture of components will be more Gaussian. Reciprocally, the individual signals will be independent if we break the Gaussian observation down into a set of nonGaussian mixtures, each with distributions that are non-Gaussian as possible.

ICA technique could be used to estimate a mixing matrix $A$ and the independent sources $X$ from a set of watermarked contents $Y$ using the matrix formulation:

$$
Y=A X
$$

While using FastICA, which is a popular ICA algorithm that achieves a fast operation and reliability of the extracted basis vector (Bas and Hurri, 2006), we are able to compute the matrices $\mathrm{A}$ and $\mathrm{X}$ and extract the estimated projection vector $\hat{p}$ located in one of the columns of the matrix A.

A limitation of ICA technique is the fact that the secret carrier could be estimated up to sign, but this will be solved by multiplying the independent components by -1 without affecting the model. Another ambiguity of ICA is that the estimated independent components may appear in an arbitrary (column) order, but one of them would be the proper estimated secret carrier.

\subsubsection{PCA Attack}

Another technique called Principal Component Analysis (PCA) could be used by an attacker to estimate the projection vector $p$ as done in (Cao, 2014). PCA identify a smaller number of uncorrelated variables known as principal components from a complex data set. It is a variable reduction procedure, which is useful to be applied on a number of redundant variables. Reducing the observed variables into a smaller number of principal components will account for most of the variance in the observed variables. Therefore, we could extract the relevant information from the complex data, and this is achieved by computing the eigenvector with the highest eigenvalue. A helpful strategy for an opponent is the Known Original Attack (KOA). In such a case, the original signal is known, and the opponent goal is to gain information about the structure of the secret key, in order to hack later on several watermarked signal. By this way, the attacker will get the quantization error $q^{\prime}$ presented in equation (5) and will try to estimate the projection vector $\hat{p}$ using PCA.

STDM spreads the distortion into a sample vector $x$, based on the projection vector $p$ as shown in equation (4). By this way, the watermark energy is focused in the projection vector after the STDM embedding. The quantization error $q^{\prime}$ as shown in equation (5), makes the variables correlated because the same projection vector $p$ is used during the embedding process. Using PCA, we are able to estimate the principal component, which is the eigenvector of the highest eigenvalue, and in our case, this principal component would be the estimated projection vector $\hat{p}$. By this way, the attacker will be able to estimate the projection vector and will get the possibility to copy, modify or remove the watermark.

As we will see in Section 4, the opponent can estimate the projection vector using PCA or ICA, therefore we applied a simple modification to the STDM watermarking scheme to overcome such kind of attacks.

\section{PROPOSED CAR-STDM METHOD}

The projection vector $p$ is an essential part of the STDM watermarking method, that is used as a secret key to embed and extract the watermark. The observation of several watermarked signals can provide sufficient information for an attacker to estimate the projection vector, by using the PCA or ICA attacks. The proposed scheme takes into account the 
security constraint and tries to overcome such kind of BSS attacks.

\subsection{Embedding Process}

The embedding process is divided into 4 main steps as shown in Figure 1. The main idea is to have a number of projection vectors equal to the number of the bits to be embedded.

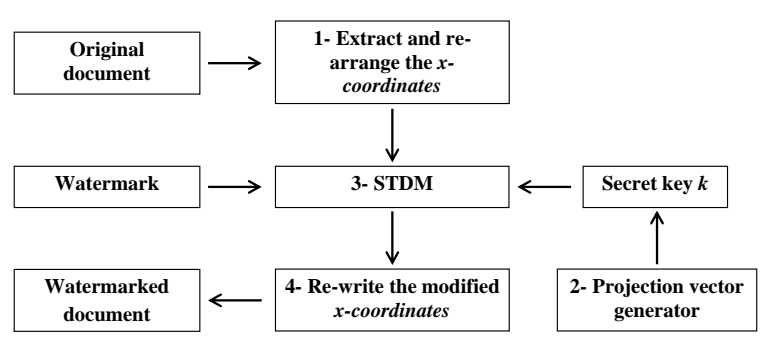

Figure 1: Embedding Block diagram.

The first step consists of reading the original document, extracting the $x$-coordinates $c_{i}$ of the existing characters and rearranging them into a matrix $X$ of size $L \times N$. Each character in the PDF document is located horizontally and vertically based on the coordinate pair $(x, y)$. The $x$-coordinates values are nonconstant, therefore they are exploited as the watermarking space in order to embed the watermark.

For the second step, we use one secret key $k$ shared between the embedder and decoder as a seed of a cryptographically secure pseudorandom number generator (PRNG) to produce $L$ projection vectors. This step is further denoted as "Projection vector generator" and is illustrated in Figure 2.

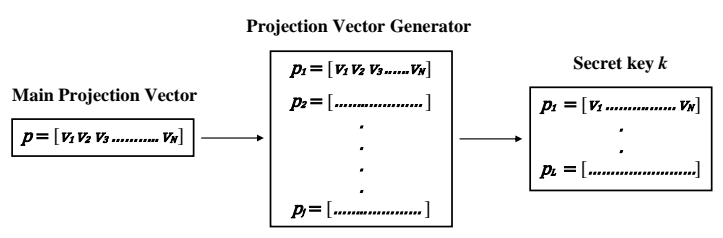

Figure 2: Projection Vector Generator.

The third step consists of embedding each bit of the watermark into the $x$-coordinates values using equation (4). We will use one projection vector per embedded bit, for that we only have to replace $p$ with $p_{i}$ in equation (4).

The last step consists of rearranging and re-writing the modified $x$-coordinate of each character into the watermarked document.

e.g. Assume that $\mathbf{b}$ of length $L$ is the binary representation of the secret message. Each bit of $\mathbf{b}$ will be em- bedded into the host signal $X$ using a projection vector $p_{i}$ of length $N$. For instance, suppose that we have a

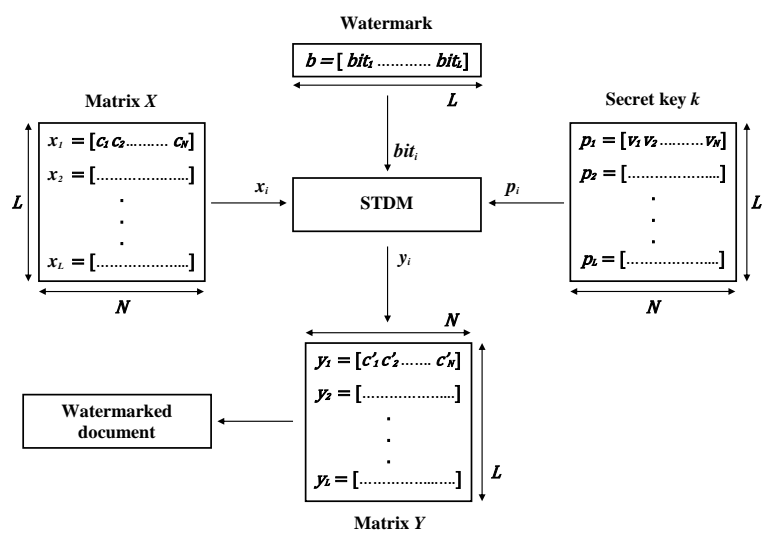

Figure 3: Embedding the $\mathrm{i}^{\text {th }}$ bit of the secret message into a set of $x$-coordinates $x_{i}$ using a projection vector $p_{i}$.

PDF file containing 800 characters through which we will embed a watermark of length $L=24$ bits. If we consider that the length of projection vector $N=8$; in this case, we need 192 characters $(24 \times 8)$ to embed the watermark. Hence 192 characters will be selected from the original document and the $x$-coordinates will be rearranged into a matrix $X$. Using the secret key $k$, 24 projection vectors will be extracted from the projection vector generator and each bit will be projected into $8 x$-coordinates using different projection vector. This technique is supposed to enhance the security of the traditional STDM, and hence nullify the effect of the PCA and ICA attacks.

\subsection{Extraction Process}

The extraction process is divided into 2 main steps as shown in Figure 4.

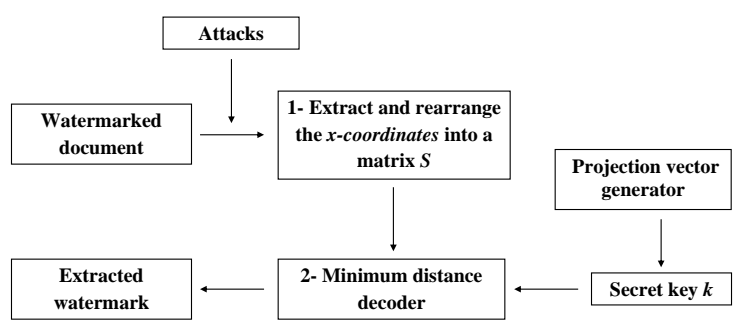

Figure 4: Extraction Block diagram.

The first step consists of reading the watermarked document and extracting the $x$-coordinates of the existing characters and rearranging them into a matrix $S$ of size $L \times N$. Matrix $S$ is the watermarked matrix $Y$ subject to various attacks.

The second step consists of extracting the embedded message by using the minimum distance decoder, 
based on the secret key $k$ as of the form:

$$
\hat{m}_{i}=\underset{m \in\{0,1\}}{\operatorname{argmin}}\left|s^{T} p_{i}-Q_{m}\left(s^{T} p_{i}, \Delta\right)\right|
$$

where $s$ corresponds to a vector of $x$-coordinates of length $N$, and $p_{i}$ represents the projection vector, used to extract the $i^{t h}$ bit of the secret message.

\section{EVALUATION OF THE PROPOSED APPROACH}

In this section, we compare the performance of the traditional STDM watermarking scheme and the proposed CAR-STDM watermarking scheme in terms of security (Section 4.1), transparency (Section 4.2), and robustness (Section 4.3). In a practical point of view, same embedding parameters have been used such as the length of the host signal and the secret message, dither level, and quantization step. All the experiments were conducted while varying the quantization step $\Delta$, and the simulations were repeated 500 times. The embedded PRNG is BSS (Blum et al., 1986), Blum Blum Shub as an instance of cryptographically secure PRNG.

\subsection{Security}

PCA and ICA have been used to compare the security level of the traditional STDM watermarking scheme and the proposed CAR-STDM watermarking scheme. The security level is measured by comparing the Bit Error Rate (BER) of the extracted message to the original one after the estimation of the projection vector used at the encoder using the PCA and ICA attacks. Experiments are done while modifying the length of the embedded message from 200 to 2000 bits, and the length of the projection vector from 8 to 32 . The original document chosen during the experimental tests contains 64000 characters. BER has been used as the error measurement between the original watermark $m$ and the extracted watermark $\hat{m}$. If the BER tends to 0 , means that the extracted watermark is similar to the original one, which means that the opponent gets an accurate estimation of the projection vector.

Figure 5 and Figure 6 show that the CAR-STDM achieves a high level of security, where the BER of the extracted watermark is close to a random guess of 0.5. This high security level is due to the fact that multiple projection vectors are used during the embedding process instead of one, which nullifies the effect of PCA and ICA attacks to estimate the projection vectors, and prevents the opponent to copy, modify or remove the watermark.

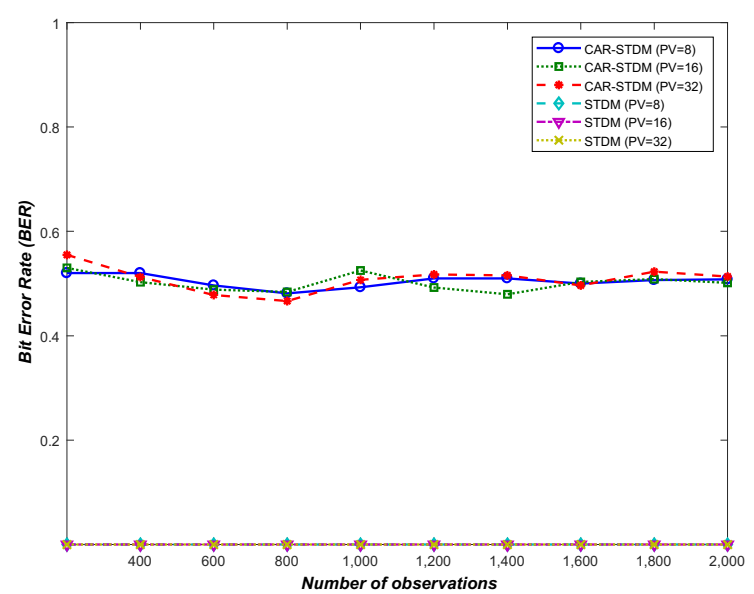

Figure 5: The comparison between the proposed CARSTDM and the traditional STDM while modifying the length of the projection vector from 8 to 32 and the number of observations from 200 to 2000, while applying the ICA attack.

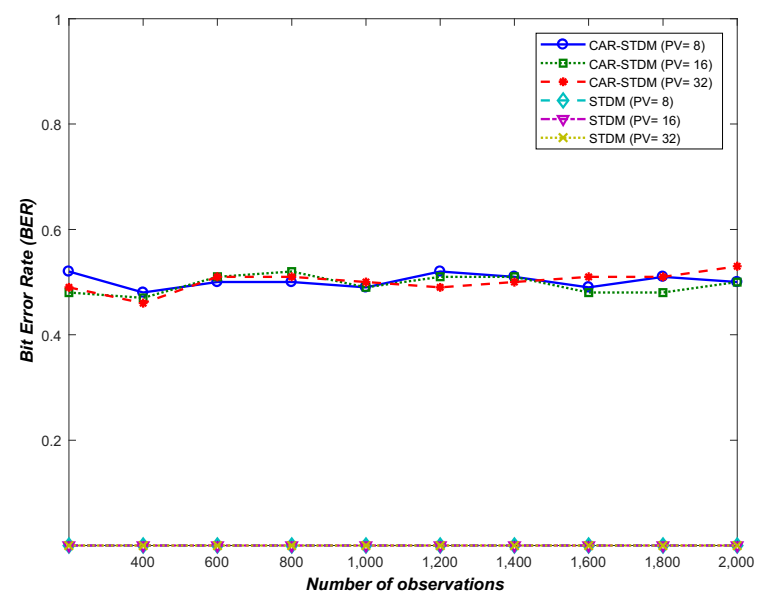

Figure 6: The comparison between the proposed CARSTDM and the traditional STDM while modifying the length of the projection vector from 8 to 32 and the number of observations from 200 to 2000, while applying the PCA attack.

In the traditional STDM method, a single projection vector is used during the embedding process, which decreases the security level. PCA and ICA can exactly estimate the projection vector due to the fact that the watermark energy is focused in the projection vector after the STDM embedding. Even if we vary the length of projection vector, the BER of the extracted watermark always tends to 0 , which means that the opponent will get an accurate estimation of the projection vector through the PCA and ICA attacks. With the CAR-STDM, a unique projection vector $p_{i}$ is generated to embed each $i^{\text {th }}$ bit of the watermark, which will makes the watermarked vec- 
Table 1: Distortin values when applying STDM and CARSTDM.

\begin{tabular}{|l|c|c|c|}
\hline$\Delta$ & $\mathrm{D}_{s}$ & STDM (MSE) & CAR-STDM (MSE) \\
\hline 0.1 & 0.00002 & $2.2444 \times 10^{-5}$ & $2.3127 \times 10^{-5}$ \\
\hline 0.5 & 0.00053 & $6.3574 \times 10^{-4}$ & $5.9178 \times 10^{-4}$ \\
\hline 1 & 0.00214 & 0.0023 & 0.0020 \\
\hline 1.5 & 0.00481 & 0.0058 & 0.0067 \\
\hline 2 & 0.08550 & 0.0090 & 0.0087 \\
\hline 2.5 & 0.01335 & 0.0144 & 0.0150 \\
\hline 3 & 0.01923 & 0.0198 & 0.0199 \\
\hline 5 & 0.05342 & 0.0687 & 0.0665 \\
\hline 8 & 0.13675 & 0.1612 & 0.1618 \\
\hline 10 & 0.21367 & 0.1886 & 0.1739 \\
\hline
\end{tabular}

tors uncorrelated from each other, and void the effect of PCA. At the same time, we will not get any relevant information from the watermarked vectors, which will block the effect of ICA to estimate the projection vectors. Since we use a cryptographically secure PRNG for building each projection vector, any algebraic method will fail to estimate the projection vectors. The obtained practical results are coherent with this theoretical analysis.

While increasing the security of the watermarking scheme, we should preserve the efficiency in term of transparency and robustness. Therefore, we compared the CAR-STDM to the traditional STDM to make sure that the modification did not affect the efficiency of the main watermarking scheme.

\subsection{Transparency}

The transparency of the proposed CAR-STDM and the traditional STDM has been tested using several values of quantization step $\Delta$. We consider a part of the original document containing 952 characters, and a watermark message "LAW" is encoded using 8 bits per character in order to form a total of 24 bits. For that, the length of the projection vector used during the embedding and decoding concept is $N=952 / 24 \approx$ 39.

Table 1 presents the distortion plots of each value of $\Delta$, and the error measurements between the watermarked document and the original one, using the Mean Square Error (MSE) and the average expected distortion $D_{s}$ (Chen and Wornell, 2001b) presented as:

$$
D_{s}=\Delta^{2} / 12 N
$$

When $\Delta$ increases, the error values increase as well. As expected, the error values of the CAR-STDM and the Traditional STDM watermarking methods are very close to each other. As shown in Figure 7, we get
Digital networks, an essential communication mechanism, are used to transmit any sort of information such as text, audio and image. Due to the rapid growth of the Internet, access to data sets has become much easier, providing a significant number of problems such as illegal distribution, authentication, duplication and malicious tampering of digital data. Among many approaches of protection, digital watermarking is undoubtedly the one that has received the most attention and interest. The watermark carries information about the object in which it is hidden, and only becoming visible

(a) $\Delta=3$

Digital networks, an essential communication mechanism, are used to transmit any sort of information such as text, audio and image. Due to the rapid growth of the Internet, access to data sets has become much easier, providing a significant number of problems such as illegal distribution, authentication, duplication and malicious tampering of digital data. Among many approaches of protection, digital watermarking is undoubtedly the one that has received the most attention and interest. The watermark carries information about the object in which it is hidden, and only becoming visible

(b) $\Delta=5$

Digital networks, an essential communication mechanism, are used to trans mit any sort of information such as text, audio and image. Due to the rapid growth of the Internet, access to data sets has become much easier providing a significant number of problems such as illegal distribution, authentication, duplication and malicious tampering of digital data. Among many approaches of protection, digital watermarking is und oubtedly the one that has received the most attention and interest. The waterma rk ca rrie s informa tion a bout the object in which it is hidden, and only becoming visible

\section{(c) $\Delta=10$}

Figure 7: Perceptual visualization of the watermarked document using the proposed CAR-STDM for $\Delta=3, \Delta=5$ and $\Delta=10$ gradually from top to bottom.

a slight modification in the characters position when $\Delta$ is smaller or equal to 3 and a notable modification when $\Delta$ is greatest than 3 .

\subsection{Robustness}

The robustness experiments were conducted by applying the Gaussian and Salt\&Pepper watermarking attacks to the $x$-coordinates of the characters in the watermarked document with two density values $(d=$ 0.1 and $d=0.25$ ). Therefore, different robustness threshold levels are computed respectively from the experiments of each type of attacks. Only the digits after the decimal point are modified. The Bit Error Rate (BER) was computed, by comparing the original watermark to the extracted one, to make the objective performance comparison. As shown in Figure 8 and Figure 9 the values of the proposed CAR-STDM and the traditional STDM are very close to each other and achieve a higher level of robustness. The robustness increases with a higher value of $\Delta$. Figure 10 shows the robustness of the CAR-STDM versus the traditional STDM under the AWGN attack, where the strength of the AWGN attack is evaluated by mean of the Watermark to Noise ratio (WNR):

$$
W N R=10 \log _{10}\left(\frac{\sigma_{w}^{2}}{\sigma_{n}^{2}}\right)
$$




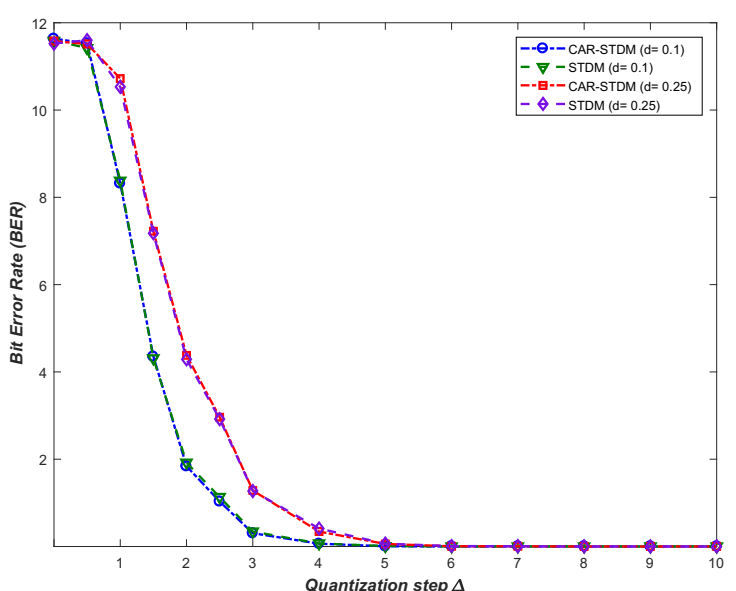

Figure 8: Comparison between the proposed CAR-STDM and the traditional STDM in terms of BER under Gaussian attack.

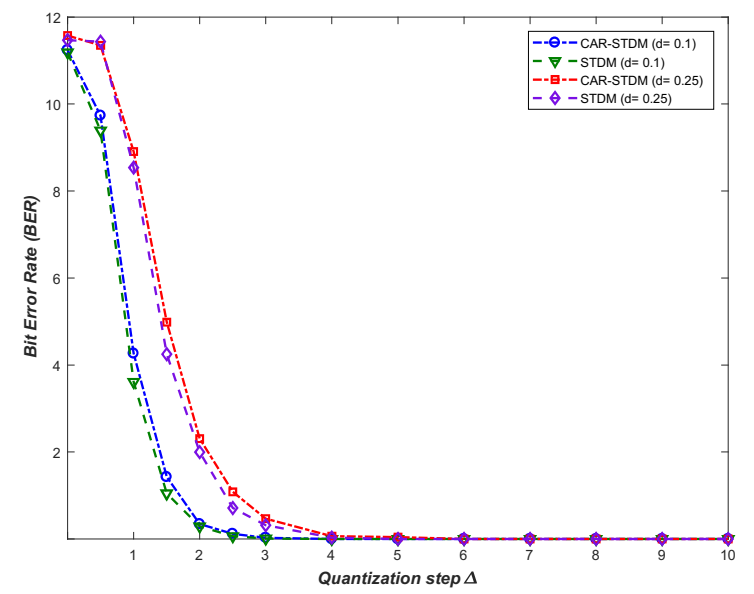

Figure 9: Comparison between the proposed CARSTDM and the traditional STDM in terms of BER under Salt\&Pepper attack.

The BER assesses the robustness level of the watermarking technique. As shown in Figure 10, for $\Delta$ equal to 2.5 , the BER is equal to 0 when the WNR is greater or equal to 10 , and the BER is greater than 0.12 when the WNR is smaller than 4 . Consequently, the robustness of the CAR-STDM and the traditional STDM increases while $\Delta$ increases.

\subsection{Our Method VS Related Work}

(Cao, 2014) proved that an attacker could estimate the projection vector of STDM, for that they proposed an improved method called ISTDM. In this method, the host signal is divided into two mutually orthogonal subspaces with the same dimension. The first one is called a reference subspace, and the other one

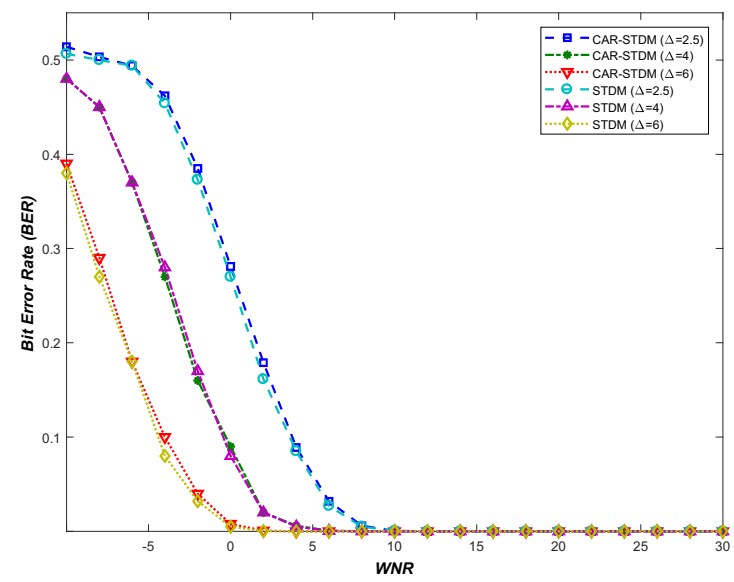

Figure 10: The robustness of the proposed CAR-STDM to that of STDM against the AWGN while varying the WNR.

is called an embedding subspace. ISTDM embed the watermark only in the embedding subspace using the STDM algorithm, in which the projection vector $p$ is the projection of the normalized host signal onto the reference subspace. This method has been tested using a Gaussian-distributed with mean vector 0 as a host signal. In addition to that, the capacity has decreased, and only the half size of the host signal could be used to embed the watermark.

In contrast, our proposed watermarking scheme embeds the watermark in a PDF document for copyright protection under a sufficient transparency-robustness tradeoff while taking into account the security constraint. We exploited the $x$-coordinates values of characters as real cover elements to embed the watermark. Furthermore, CAR-STDM could not be attacked by any algebraic methods such as PCA and ICA and preserved the capacity of STDM, therefore the watermark could be embedded in all the cover elements.

\section{CONCLUSION AND FUTURE WORK}

In this position paper, we have presented the Component Analysis Resistant-STDM watermarking scheme. Unlike the traditional STDM watermarking, the CAR-STDM uses multiple projection vectors at the encoder and the decoder. The simple but effective idea is to produce one projection vector per an embedded bit thanks to a cryptographically secure PRNG, whose seed is a key shared between the encoder and the decoder.

The $x$-coordinates values of character PDF elements have been used as cover elements to embed the watermark in this article, but any element can be used 
as support to contain the mark. Theoretically speaking, any algebraic attack such as PCA and ICA fails with this proposal. The experimental results confirm that the CAR-STDM approach achieves the security against such kind of BSS attacks, with higher level of transparency and robustness against AWGN and Salt\&Peper attacks.

As for future enhancements, we plan to execute the theoretical proof of the security analysis and to include further improvements by exploring new secure subspaces to embed the watermark.

\section{ACKNOWLEDGEMENTS}

This work is partially funded with support from the National Council for Scientific Research in Lebanon CNRS-L, the Hubert Curien CEDRE programme, the Agence Universitaire de la Francophonie AUF-PCSI programme, and the Labex ACTION program (contract ANR-11-LABX-01-01).

\section{REFERENCES}

Alizadeh-Fahimeh, F., Canceill-Nicolas, N., DabkiewiczSebastian, S., and Vandevenne-Diederik, D. (2012). Using steganography to hide messages inside pdf files. SSN Project Report.

Bas, P. and Hurri, J. (2006). Vulnerability of dm watermarking of non-iid host signals to attacks utilising the statistics of independent components. IEE Proceedings-Information Security, 153(3):127-139.

Bitar, A. W., Darazi, R., Couchot, J.-F., and Couturier, R. (2017). Blind digital watermarking in pdf documents using spread transform dither modulation. Multimedia Tools and Applications, 76(1):143-161.

Blum, L., Blum, M., and Shub, M. (1986). A simple unpredictable pseudo-random number generator. SIAM Journal on computing, 15(2):364-383.

Cao, J. (2014). Improved spread transform dither modulation: a new modulation technique for secure watermarking. In International Workshop on Digital Watermarking, pages 399-409. Springer.

Chen, B. and Wornell, G. W. (2001a). Quantization index modulation: A class of provably good methods for digital watermarking and information embedding. IEEE Transactions on Information Theory, 47(4):1423-1443.

Chen, B. and Wornell, G. W. (2001b). Quantization index modulation methods for digital watermarking and information embedding of multimedia. Journal of VLSI signal processing systems for signal, image and video technology, 27(1-2):7-33.

Cox, I., Miller, M., Bloom, J., Fridrich, J., and Kalker, T. (2007). Digital watermarking and steganography. Morgan Kaufmann.
Cox, I. J., Kilian, J., Leighton, T., and Shamoon, T. (1996). Secure spread spectrum watermarking for images, audio and video. In Image Processing, 1996. Proceedings., International Conference on, volume 3, pages 243-246. IEEE.

Hyvarinen, A. (1999). Fast and robust fixed-point algorithms for independent component analysis. IEEE transactions on Neural Networks, 10(3):626-634.

Hyvärinen, A., Karhunen, J., and Oja, E. (2004). Independent component analysis, volume 46 . John Wiley \& Sons.

Iso, T. (2008). 171/sc 2: Iso 32000-1: 2008 document management-portable document format-part 1: Pdf 1.7 .

Kuribayashi, M., Fukushima, T., and Funabiki, N. (2017). Data hiding for text document in pdf file. In International Conference on Intelligent Information Hiding and Multimedia Signal Processing, pages 390-398. Springer.

Lee, I.-S. and Tsai, W.-H. (2010). A new approach to covert communication via pdf files. Signal processing, 90(2):557-565.

Lin, H.-F., Lu, L.-W., Gun, C.-Y., and Chen, C.-Y. (2013). A copyright protection scheme based on pdf. Int $J$ Innov Comput Inf Control, 9(1):1-6.

Por, L. Y. and Delina, B. (2008). Information hiding: A new approach in text steganography. In WSEAS International Conference. Proceedings. Mathematics and Computers in Science and Engineering, number 7. World Scientific and Engineering Academy and Society.

Wang, C. and Tsai, W. (2008). Data hiding in pdf files and applications by imperceivable modifications of pdf object parameters. In Proceedings of 2008 Conference on Computer Vision, Graphics and Image Processing, Ilan, Taiwan, Republic of China. Asia University. 